\section{POS0551 MEDICAL COSTS FOR PATIENTS STARTING TREATMENT FOR RHEUMATOID ARTHRITIS WHO HAVE COMORBID DIABETES MELLITUS IN JAPAN}

E. Tanaka ${ }^{1}$, E. Inoue ${ }^{1,2}$, R. Sakai ${ }^{1}$, I. Katsuhiko ${ }^{3}$, A. Shoji ${ }^{3}$, M. Harigai ${ }^{1}{ }^{1}$ Tokyo Women's Medical University School of Medicine, Department of Rheumatology, Tokyo, Japan; ${ }^{2}$ Showa University, Showa University Research Administration Center (SURAC), Tokyo, Japan; ${ }^{3}$ Medilead Inc., Tokyo, Japan

Background: Rheumatoid arthritis (RA) patients can experience various comorbidities ${ }^{1}$. The incidence of diabetes mellitus (DM) is reported higher in patients with $\mathrm{RA}^{2}$ and comorbid DM is likely to affect treatment outcomes ${ }^{3}$ and then healthcare resource uses, however, no previous study has not focused on it. Objectives: To evaluate medical costs and resource use in patients starting treatment for RA with and without DM using a large claims database in Japan. Methods: We used a large Japanese administrative claims database constructed by the Japan Medical Data Center (JMDC) ${ }^{4}$. Patients with the International Classification of Diseases 10th revision (ICD-10) codes for RA who started medication with disease-modifying antirheumatic drugs (DMARDs) after 6 months without them in the period from 1/1/2012 to 12/31/2017 and who were observable for 12 months as a follow-up period were enrolled. These patients were categorized as DM or non-DM group with ICD-10 codes for DM plus use of antidiabetic drugs in 6 months before starting DMARDs (baseline period). To adjust baseline characteristics between the 2 groups, they were matched by sex, age, Charlson Comorbidity Index (CCl) except for DM, months from the first RA codes to starting DMARDs, and medications. The primary endpoint was mean medical cost per patient in the 12-month follow-up period. Costs in JPY were converted into EUR (1 EUR = $125 \mathrm{JPY}$ in 2020). Costs for drugs, treatments, and materials and their subcategories were evaluated both with and without DM-specific costs. The secondary endpoints were the proportions of patients using the subcategories of each resource.

Results: Patients of 161 for the DM group and 2,974 for the non-DM group were eligible, and 109 patients were matched from each group. The medians of age and $\mathrm{CCl}$ were 59 years and 2.0 in both groups and no significant difference was observed in all baseline characteristics used for matching between the groups. Total mean costs were significantly higher in the DM group (DM, 5,331 EUR, non-DM 3,200 EUR; $P<0.05)$. After excluding DM-specific costs, drug costs were significantly higher in the DM group than in the non-DM group (DM 1,883 EUR, non-DM 896 EUR; $P<0.05$ ), especially costs for biological DMARDs (DM 1,156 EUR, non-DM 292 EUR; $P<0.05$ ), mainly because a higher proportion of patients used these drugs in the DM group (Table 1). Treatment costs (DM 2,380 EUR, non-DM 2,133 EUR) and material costs (DM 74 EUR, non-DM 149 EUR) were not different between the groups, but only costs for examinations were significantly higher in the DM group (DM 970 EUR, non-DM 779 EUR; $P<0.05$ ).

Table 1. Number and proportion of patients who used drugs

\begin{tabular}{lcccc}
\hline Type of drug & & \multicolumn{3}{c}{ Drug use, $\mathrm{n}(\%)$} \\
\cline { 3 - 5 } & & DM $(\mathrm{N}=109)$ & Non-DM $(\mathrm{N}=109)$ & $P$-value \\
\hline \multirow{2}{*}{ csDMARDs } & $109(100.0)$ & $109(100.0)$ & 1.000 \\
& Total & $101(92.7)$ & $102(93.6)$ & 1.000 \\
bDMARDs & Methotrexate & $46(42.2)$ & $51(46.8)$ & 0.583 \\
& Others & $16(14.7)$ & $6(5.5)$ & 0.041 \\
& Total & $11(10.1)$ & $4(3.7)$ & 0.118 \\
& TNFi & $6(5.5)$ & $2(1.8)$ & 0.219 \\
tsDMARDs & IL6i & $4(3.7)$ & $0(0.0)$ & 0.125 \\
CSs & T-cell & $0(0.0)$ & $0(0.0)$ & 1.000 \\
Analgesics & & $65(59.6)$ & $62(56.9)$ & 0.711 \\
& Total & $103(94.5)$ & $96(88.1)$ & 0.167 \\
& Acetaminophen & $24(22.0)$ & $23(21.1)$ & 1.000 \\
& Acetaminophen /Opioids & $10(9.2)$ & $6(5.5)$ & 0.454 \\
& NSAIDs & $102(93.6)$ & $93(85.3)$ & 0.093 \\
& Opioids & $0(0.0)$ & $4(3.7)$ & 0.125 \\
& Others & $25(22.9)$ & $17(15.6)$ & 0.185
\end{tabular}

bDMARDs=biological disease-modifying antirheumatic drugs; CSs=corticosteroids; csDMARDs=conventional synthetic disease-modifying antirheumatic drugs; DM=diabetes mellitus; IL6i=interleukin-6 inhibitor; NSAID=non-steroidal anti-inflammatory drug; T-cell=selective T-cell co-stimulation modulator; TNFi=tumor necrosis factor $\alpha$ inhibitor; tsDMARDs=targeted synthetic disease-modifying antirheumatic drugs; P-values were calculated using McNemar test

Conclusion: Medical costs for RA were higher in the DM group than in the non-DM group because of more prevalent use of biological DMARDs in the DM group. REFERENCES:

[1] Gabriel SE et al., Arthritis Res Ther. 2009;11(3):229.

[2] Giacomelli R et al., Expert Rev Clin Immunol. 2016;12(8):849-55.

[3] Crepaldi G et al., PLoS One. 2016;11(1):e0146991.

[4] JMDC claims database, Tokyo, Japan.

Disclosure of Interests: Eiichi Tanaka Speakers bureau:AbbVie GK, Asahi Kasei

Pharma Corporation, Astellas Pharma Inc, Ayumi Pharmaceutical Corporation,
Chugai Pharmaceutical Co., Ltd., Eisai Co., Ltd., Eli Lilly Japan K.K., GlaxoSmithKline K.K., Kyowa Pharma Chemical Co., Ltd., Janssen Pharmaceutical K.K., Mochida Pharmaceutical Co., Ltd., Pfizer, Takeda Pharmaceutical Co., Ltd and Teijin Pharma Ltd., Eisuke Inoue Speakers bureau: Pfizer Japan, Bristol-Myers Squibb K.K., Ryoko Sakai Speakers bureau: Bristol Myers Squibb Co., Ltd. Grant/research support from: Tokyo Women's Medical University (TWMU), particularly the Division of Multidisciplinary Management of Rheumatic Diseases, Department of Rheumatology, has received unrestricted research grants from Ayumi Pharmaceutical Co.; Chugai Pharmaceutical Co., Ltd.; Eisai Co., Ltd. Nippon Kayaku Co., Ltd.; Taisho Toyama Pharmaceutical Co., Ltd.; Takeda Pharmaceutical Co., Ltd.; Mitsubishi Tanabe Pharma Co.; and Teijin Pharma Ltd., with which TWMU paid the salaries of RS., Iwasaki Katsuhiko: None declared, Ayako Shoji: None declared, masayoshi harigai Speakers bureau: AbbVie GK, Ayumi Pharmaceutical Corporation, Bristol-Myers Squibb K.K., Chugai Pharmaceutical Co., Ltd., Eisai Co., Ltd., Eli Lilly Japan K.K., Pfizer Japan Inc., and Takeda Pharmaceutical Co., Ltd., Consultant of: AbbVie GK, Bristol-Myers Squibb K.K. Chugai Pharmaceutical Co., Ltd., Eli Lilly Japan K.K., and Gilead Sciences Inc., Grant/research support from: AbbVie GK, and Asahi Kasei Corp., Astellas Pharma Inc., Ayumi Pharmaceutical Corporation, Bristol-Myers Squibb K.K. Chugai Pharmaceutical Co., Ltd. Daiichi-Sankyo, Inc., Eisai Co., Ltd., Mitsubish Tanabe Pharma Corporation., Nippon Kayaku Co., Ltd., Taisho Pharmaceutical Co., Ltd., and Takeda Pharmaceutical Co., Ltd.

DOI: 10.1136/annrheumdis-2021-eular.2770

\section{POS0552 DIASTOLIC DYSFUNCTION ASSOCIATED WITH INCREASED ACTIVITY IN RHEUMATOID ARTHRITIS}

S. Lugo-Perez¹ , D. Á. Galarza-Delgado² ${ }^{2}$, J. R. Azpiri-López¹, I. J. ColungaPedraza $^{2}$, N. Guajardo-Jauregui ${ }^{2}$, A. B. Rodriguez-Romero ${ }^{2}$, A. Cárdenas ${ }^{2}$, H. Azpiri-Diaz" ${ }^{1}$ O. A. Cepeda-Ayala ${ }^{1}$. ${ }^{1}$ Hospital Universitario "Dr. José Eleuterio González", Universidad Autónoma de Nuevo León, Cardiology, Monterrey, Mexico; ${ }^{2}$ Hospital Universitario “Dr. José Eleuterio González", Universidad Autónoma de Nuevo León, Rheumatology, Monterrey, Mexico

Background: Cardiovascular disease (CVD) is the main cause of mortality in patients with rheumatoid arthritis (RA) reflected by a higher prevalence of cardiovascular risk factors (CVRFs), a chronic systemic inflammatory state and heart failure compared to the general population (1). Left ventricular diastolic dysfunction (LVDD) is attributable to structural abnormalities such as hypertrophy or interstitial fibrosis and impaired myocyte relaxation resulting from ischemia and is frequently asymptomatic (2). The presence of LVDD it could be considered as the first step to development of heart failure.

Objectives: The aim of the study is to identify the association of disease activity and the presence of LVDD in patients with RA.

Methods: A cross-sectional, observational, and comparative study of RA subjects that fulfilled ACR / EULAR 2010 classification criteria, aged 40-75 years. Subjects were evaluated by a transthoracic echocardiogram performed and reviewed by two certified echocardiographers. A total of fifty-one RA patients diagnosed with LVDD were included according to the 2016 American Society of Echocar diography (ASE). Distribution was evaluated with the Kolmogorov-Smirnov test. Descriptive analysis was done using measures of central tendency. Chi square Student's t test and Mann-Whitney U test were used for comparations between groups. A $p$ value $<0.05$ was considered statistically significant.

Results: We found no statistical difference between groups regarding age, gender, comorbidities (type 2 diabetes mellitus, hypertension, dyslipidemia and, active smoking) and body mass index. Patients with LVDD demonstrated a higher disease activity evaluated by disease activity score using 28 joints-C reactive protein (DAS28-PCR) (4.88 vs 3.56, $p=0.004$ ) (Table 1). It was observed that patients with LVDD have a higher prevalence of being in the high disease activity category (41.2\% vs. $13.7 \%, p=0.002$ ) (Figure 1). When performing a binary logistic regression, it was found that a high disease activity was the only independent predictor for the presence of LVDD, with an OR 4.70 , $(95 \% \mathrm{Cl} 1.63-13.50, p=0.004)$.

Table 1. Demographic and disease characteristics

\begin{tabular}{lccc}
\hline & $\begin{array}{c}\text { RA patients with } \\
\text { LVDD }(\mathbf{n = 5 1 )}\end{array}$ & $\begin{array}{c}\text { RA patients without } \\
\text { LVDD }(\mathbf{n}=\mathbf{5 1})\end{array}$ & $\boldsymbol{p}$ \\
\hline Women, $\mathrm{n}(\%)$ & $50(98)$ & $47(92.2)$ & $\mathrm{NS}$ \\
Age, years \pm SD & $56.12 \pm 8.76$ & $53.91 \pm 5.61$ & $\mathrm{NS}$ \\
HTN, $\mathrm{n}(\%)$ & $17(33.3)$ & $13(25.5)$ & $\mathrm{NS}$ \\
T2DM, $\mathrm{n}(\%)$ & $6(11.8)$ & $10(19.6)$ & $\mathrm{NS}$ \\
Dyslipidemia, $\mathrm{n}(\%)$ & $17(33.3)$ & $11(21.6)$ & $\mathrm{NS}$ \\
Active smoking, $\mathrm{n}(\%)$ & $5(9.8)$ & $5(9.8)$ & $\mathrm{NS}$ \\
BMI kg/m ${ }^{2} \pm$ SD & $28.20 \pm 4.89$ & $29.40 \pm 5.13$ & $\mathrm{NS}$ \\
Disease duration, years (p25-p75) & $10.70(5.16-17.87)$ & $5.66(2.67-15.64)$ & $\mathbf{0 . 0 3 3}$ \\
DAS28-CRP, median (p25-p75) & $4.88(3.53-5.45)$ & $3.56(3.00-4.69)$ & $\mathbf{0 . 0 0 4}$
\end{tabular}

NS, no significative; HTN, hypertension; T2DM, type 2 diabetes mellitus; BMI, body mass index; DAS28, disease activity score using 28 joints; CPR, $C$ reactive protein 